\title{
Bypass Boundary Layer Transition on Flat Plate by Adverse Pressure Gradient
}

\author{
Vladislav Skála ${ }^{1}$, Václav Uruba ${ }^{1}$, Pavel Antoš ${ }^{1}$, Pavel Jonáś ${ }^{1}$ \\ ${ }^{1}$ Institute of Thermomechanics of the CAS, v. v. i. Dolejškova 1402/5 18200 Praha 8, Czech Republic
}

\begin{abstract}
Bypass boundary layer transition in flows on flat plate by adverse pressure gradient was investigated experimentally. It was measuered cases with combination of adverse pressure gradient by different free stream turbulence intenzity. Hot wire anemometry technique was used. Measuerement were made on flat plate in closed wind tunnel. Adverse pressure gradient was set by diffuser in tested section of wind tunnel. Grid turbulence of free stream was controlled by screen. Hot wire anemometry technique was used, intermitency factor was evaluated. Results were compared wih cases with simpliest conditions.
\end{abstract}

\section{Introduction}

Results of data analysis of experimental investigation of bypass transition of the laminar boundary layer to turbulence by adverse pressure gradient of main flow (using diffuser), see Fig. 1, are presented. Different cases with other boundary layer conditions were investigated.

It was measured cases with combination of roughness of surface by different free stream turbulence intenzity. This combination of conditions is usually common in real flow tasks. Better understanding and description of effect of an adverse pressure gradient and another conditions like roughness of surfaces or turbulence of external flow on the bypass transition, location and size of transition zone by transition of laminar boundary layer to turbulence, seems to be important.

Hot wire anemometry is a method for instantaneous velocity measurement.

The distributions of an intermittency factor $\gamma$ and determination of onset and terminating of transition zone are presented in this work.

\section{Experiments}

\subsection{Experimental setup}

The experiments were carried out in the closed type wind tunnel and the investigated boundary layers were developing on the smooth or rough flat plate. The orthogonal coordinate see Fig. 1.

Cross section of the test section of the wind tunnel was $0.9 \mathrm{~m} \times 0.5 \mathrm{~m}$. The length of a flat plate was of 2.65 $\mathrm{m}$. Flat plates with different roughness of surface can be changed (aerodynamically smooth and sandpaper roughness P32 and P60 particles $/ \mathrm{cm}^{2}$ ). The flow acceleration begins about $1.2 \mathrm{~m}$ upstream the flat plate.

The flow deceleration starts at a leading edge of flat plate $(x=0)$ where the flow velocity is set to $U_{\mathrm{e}}=10$ $\mathrm{m} / \mathrm{s}$. The plane diffuser had a length of $1.4 \mathrm{~m}$ and opening angle was $5^{\circ}$. It was checked carefully that there was no flow separation on the deflected wall.

The free stream turbulence was controlled by square plane mesh (grid) with cylindrical rods and square mesh holes. The free stream turbulence intensity $I u_{\mathrm{e}}$ at the leading edge was naturally $I u_{\mathrm{e}}=0.005$ or set by square grid to $I u_{\mathrm{e}}=0.03$.

A diffuser $5^{\circ}$ was placed in the test section for make the adverse pressure gradient in main flow belong flat plate. The leading edge of the plate had a super-ellipse shape MSE6.

The details of experimental settings and measurement procedures are described in $[1,2]$.

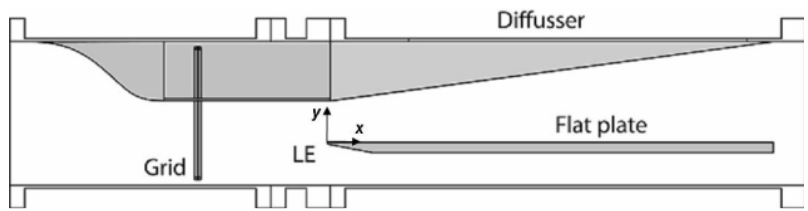

Fig. .1 Experimental setup - flat plate with diffusser along the model of flat plate in a test section.

\subsection{Measurement techniques}

Characteristics inside the boundary layer were studied by constant temperature anemometer technique. Hot wire anemometry equipment was CTA system Dantec Streamline. Time of measurement was set to $20 \mathrm{~s}$.

The hot-wire probe was put into defined positions by the computer controlled traversing device. The distance $y$ from the wall was measured by cathetometer with accuracy $\pm 0.02 \mathrm{~mm}$.

The reference value of the free stream velocity was measured by a Prandtl pressure probe (diameter $6 \mathrm{~mm}$ ) at the inlet of test section. Prandtl probe signal and

\footnotetext{
$\overline{{ }^{*} \text { Corresponding author: antos@it.cas.cz }}$
} 
barometric pressure was connected to pressure transducer Druck DPI 145 (range $7 \mathrm{kPa}$, accuracy $\pm 0.005 \%$ FS). The flow temperature was measured by thermometer Pt100. Output voltage from the transducers were read by Data acquisition unit HP 34970A.

A single hot-wire probe was used for HWA measurement. A sensor of the probe was tungsten wire. Diameter of the wire was $d_{w}=5 e-06 m$ and length of the wire was $l_{\mathrm{w}}=1.25 \mathrm{e}-03 \mathrm{~m}$. Operating wire temperature during measurement was set $T_{w}=493 \mathrm{~K}$. Hot wire sensor was oriented parallel to leading edge of flat plate. The output anemometer signal was digitalized using the A/D transducer National Instruments data acquisition system and recorded by the PC using the LabVIEW scripts (sampling frequency $75 \mathrm{kHz}, 16 \mathrm{bit}, 2.25 \mathrm{e} 6$ samples).

A cooling law for heated sensor of Koch and Gartshore (1972), was used for hot-wire measurements. Data analysis TERA method (see below) was performed using the LabVIEW scripts, results were organized using MS EXCEL.

\subsection{Measurement uncertainties}

The experimental uncertainties $\Delta$ are estimated based on calculated root mean square errors of interpolations and observed repeatability. The upper limits of relative errors of evaluated quantities depend mainly on precision of location of the probe (the distance y from surface of flat plate) and accuracy of hot-wire local instantaneous velocity $U$ measurement:

$$
\begin{aligned}
& \Delta U / U= \pm 0.005 ;\left(U_{\mathrm{e}}=10 \mathrm{~m} / \mathrm{s}\right) \\
& \Delta \mathrm{y}=0,001 \mathrm{~m} ; \\
& \Delta H_{12} / H_{12}= \pm 0.01 \\
& \Delta \gamma / \gamma= \pm 0.2
\end{aligned}
$$

\subsection{Determination of the intermittency}

The transitional intermittency is describing the laminar to turbulence transition process development in the boundary layer along the surface of the flat plate.

Boundary layer starts from leading edge of the flat plate in stream wise direction and in specific distance from leading edge starts the process of bypass laminar to turbulent flow transition. It relates to the generation and propagation of turbulent spots, turbulent spots are detected using TERA method. The transitional intermittency factor $\gamma(x)$ is one of the key parameters in recognition of the transitional boundary layer state.

Onset of transition corresponds to minimum of its value (ideally $\gamma \approx 0$ ) and the end of transition region is indicated byl $\gamma \approx 1$. Increasing value of the intermittency coefficient upstream the transition onset could be explained by disturbances penetrated from outside the boundary layer and interactions with particless of fluid from boundary layer. [4]

The intermittency factor computed by a direct method is defined as time ratio of turbulent flow occurrence time over whole measurement time

$$
\gamma(x)=\sum_{i=1}^{N} \frac{I\left(\mathrm{x}, \mathrm{t}_{i}\right)}{N},
$$

where $I\left(x, t_{i}\right)$ is indicator function in point $x$ and time $t_{i}$, derived from digitalized instantneous velocity record captured close to the surface of flat plate in distance $\mathrm{x}$ from leading edge of the flat plate, see below and Fig. 1 Fig. 5.

Direct method TERA of determination of the intermittency factor is very effective in detecting the start and the end of transition with proper choice of parameters for signal processing and analysis. A lot of methods of determination $\gamma$ is available [4], the TERA (Turbulent Energy Recognition Algorithm) method [4] was chosen.

The TERA method consists of several consecutive steps, with modify raw hot wire signal of instantneous velocity to the criterion function, see Figure 2-5. At the first, the raw instantaneous velocity signal from CTA anemometer is filtered to $u(\mathrm{t})$ by Butterworth filter with low pass frequency $1 \mathrm{kHz}$ to eliminate noise from the signal.

The second step is derivation of the signal to the detector function $D(t)$ (Fig. 4). Time derivation of signal makes the differences of the signal time behaviour during turbulent and non-turbulent periods more recognizable. Detector function is defined as

$$
D(t)=\left|u \frac{\partial u}{\partial t}\right|
$$

where $u$ is fluctuations of the instantaneous velocity.

Sensor HWA was sensitive to stream wise velocity component and to vertical velocity component. Then the HWA signal (Fig. 2) is filtered (Fig. 3) and derived to the detector function (Fig. 4). Then is detector function smoothed to criterion function (Fig. 5). The threshold value $\mathrm{K}_{\mathrm{P}}$ is applied onto criterion function $K(t)$ (see in Fig. 5) and a result is an indicator function $I(t)$ (Figure 6 ), details are presented in [4]). The threshold value $K_{P}$ is in TERA method given

$$
\left.K_{\mathrm{P}}(x)=\mathrm{C}_{2}(u(x, t) \mathrm{d} u(x, t) / \mathrm{d} t)_{\mathrm{RMS}}\right)
$$

index RMS means the standard deviation evaluated during a time of measuerement of one datat point .

The disadvantage of TERA algorithm is well known. It is necessary manually find the "right value" of threshold constant $C_{2}$ constant in equation (2).

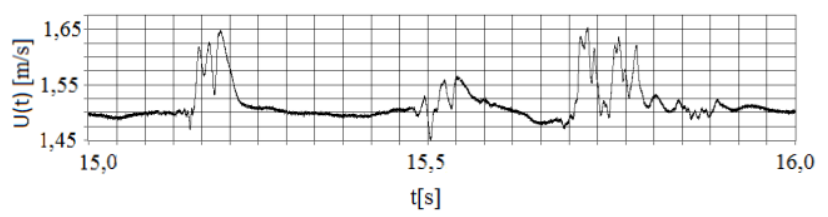

Fig. 2. Raw instantaneous velocity $\mathrm{U}(\mathrm{t}), \mathrm{CTA}$ signal in duration $0,5 \mathrm{~s},[3]$ 




Fig. 3. Filtred signal $u(t)$ of fluctuations of instantneous velocity, [3]

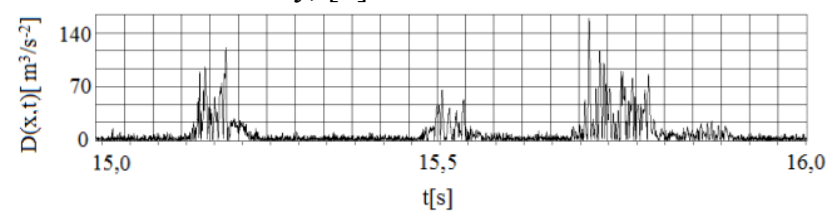

Fig. 4. Detector function $D(t)$, [3]



Fig. 5. Criterion function $\mathrm{K}(\mathrm{t})$ with threshold $\mathrm{C}=10$, [3]

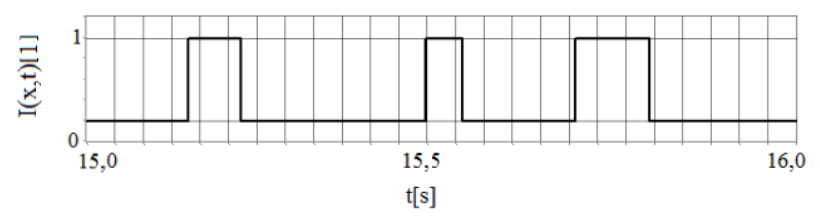

Fig. 6. Indicator function $I(t),[3]$

It was discussed different ways, how to find an optimal and objective value $C_{2}^{*}$ of threshold constant.

The most successful is a criterion using quality of interpolation of evaluated experimentally data points $\gamma$ with universal intermittency function $\gamma^{*}$ [7]

$$
\gamma^{*}(x)=1-\exp \left(-0,412\left(\frac{x-x_{0,01}}{x_{0,75}-x_{0,25}}\right)^{2}\right) \text {. }
$$

First step is calculation of a group of $\gamma_{j}^{*}$ functions for different values $C_{2 j}$ from (empirically recomended) interval

$$
C_{2 j} \in(0,05 ; 0,6),
$$

where $j=\langle 1, M\rangle, M$ is a number of iterations for evaluation $C_{2}^{*}$.

Universal intermittency function $\gamma_{j}^{*}$ for $C_{2 j}$ is expressed

$$
\gamma_{j}^{*}(x)=1-\exp \left(-0,412\left(\frac{x-x_{0,05 j}}{x_{0,75 j}-x_{0,25 j}}\right)^{2}\right),
$$

where $x_{0,25 j}$ is a point, where $\gamma_{j}\left(x_{0,25 \mathrm{j}}\right)=0,25 ; x_{0,75 \mathrm{j}}$ is a point, where $\gamma_{j}\left(x_{0,75 \mathrm{j}}\right)=0,75$, for given $C_{2 \mathrm{j}}$.
Values of $x_{0,25 \mathrm{j}}$ and $x_{0,75 \mathrm{j}}$ (for given $C_{2 \mathrm{j}}$ ) should be found using TERA method from evaluated and only locally interpolated data points of $\gamma_{\mathrm{j}}(x)$.

The correct value of $x_{0,01 \mathrm{j}}$ is very difficult to find directly from locally interpolated data points of $\gamma_{\mathrm{j}}(x)$, generally function $\gamma$ is here very flat and experimental data are usually not enough smooth. Value of $x_{0,01 \mathrm{j}}$ can be better found using another form of universal intermittency function $\gamma^{* *}(x)$, see eq. (6), [8]. This form uses experimentally data points $\gamma_{j}(x)$ from the middle part of transition zone, function $\gamma$ is here " well shaped $》$. The value of $x_{0,01 \mathrm{j}}$ is the last searched value in eq. (5)

$\gamma_{0,01 j}^{* *}(x)=0,01=1-\exp \left(-0,412\left(\frac{x_{0,05 j}-x_{0,5 j}}{x_{0,75 j}-x_{0,25 j}}+1,3\right)^{2}\right)$,

where $x_{0,5 j}$ is point, where $\gamma_{j}=0,5$. Values of $x_{0,25 \mathrm{j}}$ and $x_{0,25 \mathrm{j}}$ are known, see before.

The quality of approximation $\phi_{j}$ of of (experimentally evaluated) intermittent factor $\gamma_{j}$ with universal intermittency function $\gamma_{j}^{*}$ can be expressed (for $N$ points of $\gamma$ and $\gamma_{j}^{*}$ )

$$
\phi_{j}\left(C_{2 j}\right)=\frac{1}{N} \sum_{i=1}^{N} \sqrt{\gamma\left(x_{i}, \mathrm{C}_{2 j}\right)^{2}-\gamma^{*}\left(x_{i}, \mathrm{C}_{2 j}\right)^{2}} .
$$

Function $\phi_{j}$ depend on the (selected) constant of the threshold value $C_{2 j}$. Optimalization criterion for founding of the optimal and objective value of the treshold value $C_{2}^{*}$ can be expressed

$$
\min \left(\phi_{j}\left(C_{2 j}\right)\right) \rightarrow C_{2}^{*}
$$

It seems be capable and simple improvement of the TERA method. Now it is possible set the founded optimal value $C_{2}^{*}$ into (up to now unknown) constant $C_{2}$ in equation (2) and the objective distribution of intermittency factor $\gamma\left(C_{2}^{*}\right)$ or in the form of the universal intermittency function $\gamma^{*}$ can be evaluated.

Determination of onset and terminating of transition zone is usually covenanted. The transition onset is covenanted in $\operatorname{Re}_{2_{0,01}}$, the transition terminating is covenanted in $x_{0,99}$, where $x_{0,99 \mathrm{j}}$ is a point, where $\gamma\left(x_{0,99 j}\right)=0,99$.

Shape factor is defined

$$
H_{12}=\frac{\delta_{1}}{\delta_{2}},
$$

where $\delta_{1}$ is a displacement thickness of boundary layer and $\delta_{2}$ is a momentumt thickness of boundary layer.

The pressure gradient along the flat plate is desccribed by the ratio of the local time averaged velocity $\bar{U}_{\infty}$ and time averaged velocity $\overline{U_{\infty_{0}}}$, whre the transition process starts.

\section{Results of experiment}


The transitional intermittency represents by distribution of intermittency factor $\gamma$ evaluated for different cases using direct method TERA based on analysis of instantaneous velocity record are presented in Fig 7 - 10, threshold value problem solution by using TERA method was described in chapter 3.3.

For comparison is plotted $\gamma_{\text {IND }}$ evaluated using indirect method based on time averaged velocity profiles characteristics (for example $H_{12}$ ) based on Emmons hypothesis [9].

Intermittency factor $\gamma\left(R e_{2}\right)$ and $\gamma\left(R e_{2}\right)_{\mathrm{IND}}$, shape factor $H_{12}$ and pressure gradient distributions are plotted in Fig 7 - 10. dependently on Reynolds number $R e_{2}$ related to the momentum thickness $\delta$.

Pressure gradient distribution is expressed in a dimensionless form by a ratio of the mean velocity out of boundary layer and the mean velocity at the point, where the adversure pressure gradient starts $\overline{U_{\infty}} / \overline{U_{\infty}}$.

Region of curved surface of leading edge (»superelipticaly» shaped MSE 6) was not optically accessible for measurement of position of wire of HWA probe, separately in cases with roughness of surface.

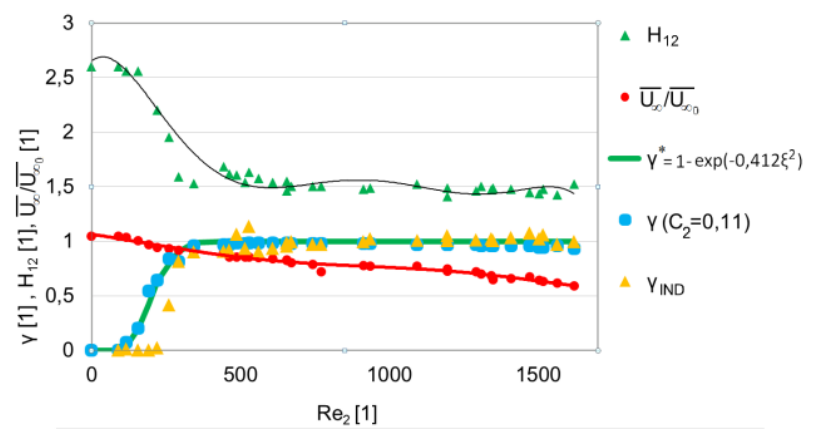

Fig. 7. $\overline{U_{\infty}} / \overline{U_{\infty}}\left(\operatorname{Re}_{2}\right), H_{12}\left(\operatorname{Re}_{2}\right), \gamma\left(\operatorname{Re}_{2}\right), \gamma\left(\operatorname{Re}_{2}\right)_{\mathrm{IND}}$, $\gamma^{*}\left(R e_{2}\right), \alpha=5^{\circ}$, smooth surface of flat plate, $I u_{\mathrm{e}}=0,5 \%$



Fig. 8. $\overline{U_{\infty}} / \overline{U_{\infty}}\left(R e_{2}\right), H_{12}\left(R e_{2}\right), \gamma\left(R e_{2}\right), \gamma\left(R e_{2}\right)_{\mathrm{IND}}$, $\gamma^{*}\left(R e_{2}\right), \alpha=5^{\circ}$ smooth surface of flat plate, $I u_{\mathrm{e}}=3 \%$

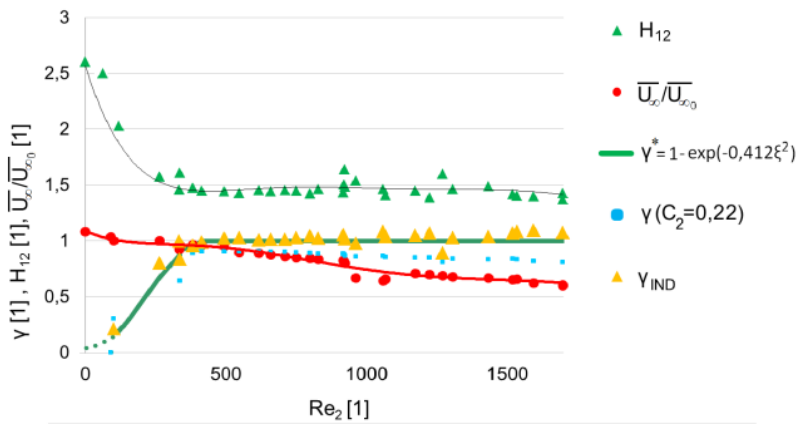

Fig. 9. $\overline{U_{\infty}} / \overline{U_{\infty}}\left(\operatorname{Re}_{2}\right), \quad H_{12}\left(\operatorname{Re}_{2}\right), \quad \gamma \quad\left(\operatorname{Re}_{2}\right), \quad \gamma\left(\operatorname{Re}_{2}\right)_{\mathrm{IND}}$, $\gamma^{*}\left(R e_{2}\right), \quad \alpha=5^{\circ}$, rough (P32) surface of flat plate, $I u_{\mathrm{e}}=0,5 \%$



Fig. 10. $\overline{U_{\infty}} / \overline{U_{\infty_{0}}}\left(\operatorname{Re}_{2}\right), \quad H_{12}\left(\operatorname{Re}_{2}\right), \quad \gamma \quad\left(\operatorname{Re}_{2}\right), \quad \gamma\left(\operatorname{Re}_{2}\right)_{\text {IND, }}$ $\gamma^{*}\left(\operatorname{Re}_{2}\right), \quad \alpha=5^{\circ}$, rough (P32) surface of flat plate, $I u_{\mathrm{e}}=3 \%$



Fig. 11. $\gamma^{*}(\zeta)$ and $\gamma(\zeta) ; \quad \zeta=\frac{x-x_{0,05}}{x_{0,75}-x_{0,25}}$ 
In Fig. 11 is ploted univerzal intermitent function $\gamma^{*}(\zeta)$ and evaluated values of $\gamma(\zeta$.).

In tables Tab. 1 and Tab. 2. are presented results of the evaluation of the covenanted transition onset in $x_{0,01}$ and $\operatorname{Re}_{2}\left(x_{0,01}\right)$ and of the covenanted transition terminating in $x_{0,99}$ and $\operatorname{Re}_{2}\left(x_{0,99}\right)$.

Table. 1. Measurement evaluation

\begin{tabular}{|c|c|c|c|c|}
\hline surface & Tu [1] & $x_{0,01}[\mathrm{~m}]$ & $x_{0,99}[\mathrm{~m}]$ & $\mathrm{C}_{2}^{*}$ \\
\hline smooth & 0,005 & 0,018 & 0,087 & 0,11 \\
\hline smooth & 0,03 & 0,042 & 0,094 & 0,21 \\
\hline rough P32 & 0,005 & 0,002 & 0,076 & 0,22 \\
\hline rough P32 & 0,03 & 0,023 & 0,068 & 0,2 \\
\hline
\end{tabular}

Table. 2. Measurement evaluation

\begin{tabular}{|c|c|c|c|c|}
\hline surface & $\mathrm{Tu}[1]$ & $\operatorname{Re}_{2 \times 0,01}[1]$ & $\operatorname{Re}_{2 \times 0,99[1]}$ & $\mathrm{C}_{2}^{*}$ \\
\hline smooth & 0,005 & 83 & 310 & 0,11 \\
\hline smooth & 0,03 & 159 & 338 & 0,21 \\
\hline rough P32 & 0,005 & 25 & 397 & 0,22 \\
\hline rough P32 & 0,03 & 87 & 405 & 0,2 \\
\hline
\end{tabular}

\section{Conclusions}

Results of experimental data analysis for cases with adversure pressure gradient are presented. The distributions of an intermittency factor $\gamma$ and determination of covenanted onset and terminating of transition zone are evaluated.

Adversure pressure gradient, in comparison with cases without adverse pressure gradient [10], naturally accelerate the transition process, it starts much closer to the leading edge of the flat plate and the length of the transitional zone is much shorted.

Higher free stream turbulence intensity and effect of and roughness of the surface are too recognizable and significant.

This work was supported by the Grant Agency of the Czech Republic, project No. 17-01088S.

\section{References}

1. P. Antoš, P. Jonáš, P. Procházka, V. Skála, V. Uruba, Effect of the Leading Edge on the Transitional Region in the Boundary Layer in External Turbulent Flow, Colloquium Fluid Dynamics 2014, p. 3-4. (2014).

2. V.Uruba, P. Jonáš, O. Mazur, Shift of the By-Pass Transition Onset due to the Change of the Incoming Turbulence Length Scale, Proceedings of Engineering Mechanics'98. 4, p.781-786. (1998).

3. O.Hladík, P.1 Jonáš, V. Uruba, Effect of the trreshold on the Determination of the Transitional Intermittency Factor, Colloquium Fluid Dynamics 2009 (2009).
4. T.B. Hedley, J.F Keffer J.Fluid Mech., 64, part 4, p. 625-644. (1974).

5. Z.Zaric, R.E.Falco, R.F. Blackwelder, Technique for the Detection of Coherent Structures in WallBounded Flows and its Application to the Analysis of MultipleWire Signals, (1984) pp. 8. 1.

6. R.E. Falco, C.P.Gendrich, Turbulence Burst Detection Algorithm of Z. Zaric,. Proc. of the Int. Centre. for Heat and Mass Transf.1988, pp. 911931.

7. R. Narasimha, The Laminar - Turbulent Transition Zone in the Boundary Layer, Prog. Aerospace Sci. Vol. 22, pp 29-80, Pergamon Press, 1985.

8. R. Narasimha, On the Distribution of Intermittency in the Transition Region of a Boundary Layer, J. Aero. Sci. 24, (9), 711-712, 1957.

9. H.W. Emons, The Laminar Turbulent Transition in Boundary Layer, Jour.Aero.Sci. 18 (1951) 490-498.

10. V. Skála, P. Antoš, O. Hladík, Intermitence factor evaluation by bypass boundary layer tranzition in flows on rough surface on flat plate, Proc. of EFM 2016, (2016). 